\title{
VALIDAÇÃO DE MÉTODO ESPECTROFOTOMÉTRICO DE ANÁLISE PARA A QUANTIFICAÇÃO DE ÁCIDO ACETILSALICÍLICO EM FORMULAÇÕES FARMACÊUTICAS: UMA PROPOSTA DE AULA EXPERIMENTAL PARA ANÁLISE INSTRUMENTAL
}

\author{
Enock José A. Goes Junior ${ }^{\mathrm{a}}$, Jakson S. Roeder ${ }^{\mathrm{a}}$, Kaiky B. L. Oliveira ${ }^{\mathrm{a}}$, Mateus P. Ferreira ${ }^{\mathrm{a}}$ e Jonatas G. da Silva ${ }^{\mathrm{b}, *}$ \\ aUniversidade Católica de Brasília, 71966-700 Taguatinga - DF, Brasil \\ ${ }^{\mathrm{b}}$ Universidade Federal do Oeste da Bahia, Campus Reitor Edgard Santos, 47810-059 Barreiras - BA, Brasil
}

Recebido em 06/08/2018; aceito em 08/10/2018; publicado na web em 29/10/2018

\begin{abstract}
VALIDATION OF A SPECTROPHOTOMETRIC METHOD FOR QUANTIFICATION OF ACETYLSALICYLIC ACID IN PHARMACEUTICAL FORMULATIONS: A PROPOSAL OF EXPERIMENTAL ACTIVITY FOR INSTRUMENTAL ANALYSIS. In the present article it is described the validation of a simple, precise, accurate, rapid and low-cost UV spectrophotometric method for the quantification of acetylsalicylic acid in pharmaceutical formulations. The method was validated by linearity, specificity, range, accuracy, precision, robustness, and limits of detection and quantification. The method was linear in the range between $24-$ $36 \mu \mathrm{g} \mathrm{mL} \mathrm{m}^{-1}$, presenting a good correlation coefficient $(\mathrm{r}=0.9994)$ and adequate limits of quantification $\left(6.57 \mu \mathrm{g} \mathrm{mL} \mathrm{m}^{-1}\right)$ and detection $\left(1.99 \mu \mathrm{g} \mathrm{mL}^{-1}\right)$. Precision analysis showed low coefficient of variation $(<2.0 \%)$ and a good average recovery percentual $(99.42 \%-$ reference and $95.94 \%$ - generic). The results and the statistical treated proved that the method may be considered a precise, accurate and low cost analytical alternative for laboratory routine and experimental activity. The method was applied for acetylsalicylic acid determination in two different pharmaceuticals formulations. The results of these analyzes are in accordance with the values declared by the manufacturer, at a 95\% confidence level. This experimental activity is a simple and might be used as an practice activities on teaching laboratories improve the student learning.
\end{abstract}

Keywords: acetylsalicylic acid; UV spectrophotometry; validation studies; pharmaceutical formulations; teaching laboratories.

\section{INTRODUÇÃO}

O ácido acetilsalicílico (AAS), quimicamente conhecido como ácido 2-acetiloxibenzóico e comumente conhecido com Aspirina ${ }^{\circledR}$ (Figura 1), é um fármaco anti-inflamatório não-esteroide utilizado para o alívio de cefaleia, inflamações e febre, apresentando propriedades antipiréticas, antirreumáticas, analgésicas e anticoagulantes. O AAS age inibindo as enzimas ciclooxigenases (COX 1 e 2), diminuindo assim, a produção de prostaglandinas, que são importantes mediadores endógenos de diversos processos fisiológicos. O AAS também é utilizado em distúrbios cardiovasculares e diabetes. ${ }^{1-3}$<smiles>CC(=O)Oc1ccccc1C(=O)O</smiles>

Figura 1. Estrutura química do ácido acetilsalicílico

Diferentes métodos analíticos como espectrofotométrico, ${ }^{4-6}$ espectrofluorimétrico, ${ }^{7,8}$ cromatográfico, ${ }^{9,10}$ potenciométrico ${ }^{11}$ e voltamétrico, ${ }^{12-14}$ são descritos na literatura para a quantificação de AAS em formulações farmacêuticas. No entanto, para o doseamento de AAS em comprimidos a farmacopeia brasileira ${ }^{15}$ descreve o método titulométrico de retorno ácido-base como oficial.

$\mathrm{Na}$ detecção e quantificação de fármacos em formulações farmacêutica a técnica espectrofotométrica na região do ultravioleta (UV) é amplamente aplicada, sendo utilizada principalmente no controle de qualidade de indústrias farmacêuticas, pois cumpre requisitos essenciais (rapidez, baixo custo operacional e confiabilidade de resultados) para as análises de rotina. ${ }^{16,17}$ São encontrados

*e-mail: jonatasg@yahoo.com.br na literatura alguns exemplos do emprego da espectrofotometria na região do UV na quantificação de fármacos como carvedilol, ${ }^{16}$ efavirenz,${ }^{17}$ sinvastatina, ${ }^{18}$ aciclovir, ${ }^{19}$ olanzapina, ${ }^{20}$ fluoxetina ${ }^{21} \mathrm{e}$ paracetamol. $^{22}$

A necessidade de proporcionar a qualidade às medições analíticas, ou seja, a confiabilidade de resultados, está sendo cada vez mais necessária e exigida. ${ }^{17}$ Portanto, métodos analíticos são constantemente validados com o objetivo de gerar resultados confiáveis. Nesse contexto, o controle de qualidade tornou-se uma ferramenta importante na indústria farmacêutica, garantindo assim um produto seguro e eficaz. ${ }^{19}$ A validação de um método analítico, para a quantificação de uma espécie química, deve assegurar por meio de estudos experimentais, que esse atenda às exigências das aplicações analíticas, assegurando a confiabilidade dos resultados. Para a validação do método analítico os seguintes parâmetros devem ser avaliados: seletividade; linearidade; intervalo, precisão, limite de detecção; limite de quantificação; exatidão; e robustez. ${ }^{23}$

Neste contexto, o presente trabalho tem como objetivo validar um método empregando a espectrofotometria na região do UV para a quantificação de AAS em formulações farmacêuticas, como uma sugestão de atividade prática para a disciplina de Análise Instrumental em cursos de graduação. Ressalta-se que a aula prática constitui uma importante ferramenta metodológica que favorece o processo de ensino-aprendizagem nas disciplinas de química. Por meio da experimentação, alia-se prática à teoria, possibilitando o desenvolvimento de habilidade manuais e intelectuais, a argumentação, a resolução de problemas, a compreensão de conceitos, podendo despertar o interesse do estudante. ${ }^{24-26} \mathrm{Na}$ literatura são encontrados alguns exemplos de propostas de atividades práticas para a disciplina de Análise Instrumental dos cursos de Química e/ou Farmácia, como a construção artesanal de um eletrodo íon seletivo a chumbo(II) enquanto alternativa para disciplinas experimentais, ${ }^{27}$ a utilização de bomba de aquário em titulações espectrofotométricas ${ }^{28}$ e a quantificação de 
ácido acetilsalicílico (AAS) em Aspirina ${ }^{\circledR}$ por titulações potenciométrica e condutométrica. ${ }^{29}$

\section{PARTE EXPERIMENTAL}

\section{Equipamentos}

As medidas espectrofotométricas no UV foram realizadas em triplicata, utilizando-se o espectrofotômetro UV-Vis Cary 50 $\left(\operatorname{Varian}^{\circledR}\right)$ de duplo feixe, detector de 190 a $1100 \mathrm{~nm}$, cubetas de quartzo de $1 \mathrm{~cm}$ e acoplado a um microcomputador. Todas as medidas de massa foram realizadas em balança analítica semi-micro ( $\sigma$ $\leq 0,05 \mathrm{mg}$ ) AUW220D $\left(\right.$ Shimadzu $\left.^{\circledR}\right)$. A solubilização do AAS em formulações farmacêuticas foi realizada com o auxilio de Ultrassom USC-1400 A (UNIQUE). A bureta de 25,00 mL utilizada na titulação de retorno ácido-base foi previamente calibrada empregando-se o método gravimétrico.

\section{Reagentes, soluções e amostras}

Todos os reagentes utilizados foram de grau analítico e as soluções foram preparadas em água ultrapura (Direct-Q ${ }^{\circledR} 8 \mathrm{UV}$ Smart, Millipore, 18,2 $\Omega$ ). O padrão de AAS $99 \%$ foi adquirido da Sigma-Aldrich (China) e utilizado sem purificação adicional. A solução estoque de AAS $1 \mathrm{mg} \mathrm{mL}^{-1}$ foi preparada dissolvendo-se 10 mg de AAS, em etanol absoluto, em balão volumétrico de $10 \mathrm{~mL}$. O hidróxido de sódio $(\mathrm{NaOH}) 97 \%$, o ácido clorídrico $(\mathrm{HCl}) 37 \%$ e o etanol absoluto $99,8 \%$ foram adquiridos da Vetec (Brasil). O biftalato de potássio $\left(\mathrm{HK}\left(\mathrm{C}_{8} \mathrm{H}_{4} \mathrm{O}_{4}\right)\right) 99,5 \%$ e o carbonato de sódio $\left(\mathrm{Na}_{2} \mathrm{CO}_{3}\right) 99,5 \%$ foram adquiridos da Merck (Alemanha) e Synth (Brasil), respectivamente. As soluções de $\mathrm{NaOH}$ foram preparadas dissolvendo-se massas adequadas desse composto em água ultrapura. As soluções de $\mathrm{HCl}$ foram preparadas por diluição em água ultrapura. Os comprimidos contendo AAS foram adquiridos em comércio local.

\section{Validação do método analítico}

O método proposto foi validado conforme a RDC $\mathrm{n}^{\circ} 166$ de 24/07/2017 da ANVISA (Agência Nacional de Vigilância Sanitária), ${ }^{23}$ que estabelece critérios para a validação de métodos analíticos empregados em insumos farmacêuticos, medicamentos e produtos biológicos em todas as suas fases de produção, e recomendações da ICH (The International Conference on Harmonisation of Technical Requirements for Registration of Pharmaceuticals for Human Use). ${ }^{30}$ Os parâmetros avaliados foram: seletividade; linearidade; intervalo, precisão, limite de detecção; limite de quantificação; exatidão; e robustez. O método foi comparado estatisticamente com o método de titulação de retorno ácido-base da Farmacopeia Brasileira. ${ }^{15}$

\section{Seletividade}

A seletividade do método foi avaliada por meio de análises dos espectros UV obtidos para o AAS e os excipientes sacarina sódica e vanilina. Foram preparadas para a sacarina sódica e vanilina soluções estoque $1 \mathrm{mg} \mathrm{mL}^{-1}$, dissolvendo-se $10 \mathrm{mg}$ da sacarina sódica ou vanilina em $\mathrm{NaOH} 0,100 \mathrm{~mol} \mathrm{~L}^{-1}$, em balão volumétrico de $10 \mathrm{~mL}$. As medidas espectrofotométricas foram realizadas nas soluções de sacarina sódica $3 \mu \mathrm{g} \mathrm{mL} \mathrm{m}^{-1}$ e vanilina $3 \mu \mathrm{g} \mathrm{mL}^{-1}$ e de uma mistura (AAS $30 \mu \mathrm{g} \mathrm{mL} L^{-1}+$ sacarina sódica $3 \mu \mathrm{g} \mathrm{mL}^{-1}+$ vanilina $3 \mu \mathrm{g} \mathrm{mL} \mathrm{m}^{-1}$ ), preparadas por diluição das soluções estoque. $\mathrm{O}$ perfil dos espectros obtidos foram avaliados e comparados com o espectro de uma solução de AAS $30 \mu \mathrm{g} \mathrm{mL}{ }^{-1}$.

\section{Linearidade e intervalo}

A linearidade foi determinada por meio da obtenção de três curvas analíticas no intervalo de AAS de 24 a $36 \mu \mathrm{g} \mathrm{mL}^{-1}$ (de 80-120\% da concentração teórica do teste).$^{23} \mathrm{~A}$ equação da reta (inclinação e intercepto com o eixo y) foi obtida pelo método dos mínimos quadrados e calculou-se o coeficiente de correlação linear. Os dados de cada nível de concentração foram avaliados por meio de análise de variância (ANOVA).

\section{Limites de detecção $(L D)$ e de quantificação $(L Q)$}

Os limites de detecção e quantificação foram calculados a partir do desvio padrão do intercepto com o eixo y $(\sigma)$ e da inclinação da curva analítica (IC), Equações 1 e $2 .^{23}$

$$
\begin{aligned}
& \mathrm{LD}=\frac{3,3 \sigma}{\mathrm{IC}} \\
& \mathrm{LQ}=\frac{10 \sigma}{\mathrm{IC}}
\end{aligned}
$$

O desvio padrão do intercepto com o eixo y foi calculado pelas seguintes Equações: ${ }^{31}$

$$
\begin{gathered}
\mathrm{S}_{\mathrm{y} / \mathrm{x}}=\sqrt{\frac{\sum\left(\mathrm{y}_{\mathrm{i}}-\hat{\mathrm{y}}\right)^{2}}{\mathrm{n}-2}} \\
\sigma=\mathrm{S}_{\mathrm{y} / \mathrm{x}} \sqrt{\frac{\Sigma \mathrm{x}_{\mathrm{i}}^{2}}{\mathrm{n} \Sigma\left(\mathrm{x}_{\mathrm{i}}-\overline{\mathrm{x}}\right)^{2}}}
\end{gathered}
$$

\section{Precisão}

A repetibilidade (precisão intradia) foi avaliada por meio de medidas espectrofotométricas de soluções contendo AAS em três níveis de concentração diferentes $\left(24,30\right.$ e $\left.36 \mu \mathrm{g} \mathrm{mL}{ }^{-1}\right)$, contemplando o intervalo linear do método, e com três réplicas cada. A precisão intermediária (precisão interdia) foi avaliada de forma semelhante à precisão intradia, no entanto, as medições espectrofotométricas foram realizadas em dois dias com analistas diferentes. Calculou-se o coeficiente de variação $(\mathrm{CV})$, por meio da Equação 5.

$$
\mathrm{CV}=\mathrm{DPR}=\frac{\mathrm{DP}}{\mathrm{CMD}} \times 100
$$

em que: CV é o coeficiente de variação; DPR é o desvio padrão relativo; DP é o desvio padrão das medidas espectrofotométricas; e CMD é a concentração média de AAS determinada.

\section{Exatidão}

A exatidão foi avaliada por meio do teste de adição de padrão de AAS, nas amostras de formulações farmacêuticas (referência e genérico), e sua recuperação em três níveis diferentes $(20 \mathrm{mg}, 50 \mathrm{mg}$ e $70 \mathrm{mg}$ ), com concentrações finais dentro da faixa de linearidade do método. Os valores de recuperação, expressos em porcentagem, foram obtidos por meio das medidas espectrofotométricas das soluções finais em função da quantidade teórica de padrão adicionado. As concentrações de AAS foram obtidas por meio da curva analítica.

\section{Robustez}

A robustez foi avaliada por meio das medições espectrofotométricas, variando-se a marca do $\mathrm{NaOH} 97 \%$ (Vetec, Reagen e Synth) e a temperatura da sala de análise $\left(27\right.$ e $\left.24{ }^{\circ} \mathrm{C}\right)$. As análises foram realizadas em triplicata e as concentrações de AAS foram obtidas por meio da curva analítica. 


\section{Quantificação de AAS em formulações farmacêuticas utilizando-se o método espectrofotométrico}

Vinte comprimidos de cada amostra comercial tiveram o seu conteúdo pesado, pulverizado e homogeneizado. Transferiu-se quantidade do pó equivalente a $0,250 \mathrm{~g}$ de AAS para béquer de $100 \mathrm{~mL}$ e em seguida adicionou-se $25 \mathrm{~mL}$ de $\mathrm{NaOH} 0,100 \mathrm{~mol} \mathrm{~L}^{-1}$. A solubilização do AAS nas amostras comerciais foi realizada com auxilio de Ultrassom por 15 minutos. Após a filtração transferiu-se $1000 \mu \mathrm{L}$ da solução resultante para balão volumétrico de $10 \mathrm{~mL}$ e completou-se o volume com $\mathrm{NaOH} 0,100 \mathrm{~mol} \mathrm{~L}^{-1}$. Em seguida transferiu-se $300 \mu \mathrm{L}$ da solução resultante para balão volumétrico de $10 \mathrm{~mL}$ e completou-se o volume com $\mathrm{NaOH} \mathrm{0,100} \mathrm{mol} \mathrm{L}^{-1}$. As análises foram realizadas em triplicata e as concentrações de AAS foram obtidas por meio da curva analítica.

\section{Quantificação de AAS em formulações farmacêuticas utilizando-se a titulação de retorno ácido-base}

A titulação de retorno ácido-base recomendada pela Farmacopeia Brasileira $^{15}$ foi aplicada como método de comparação ao método proposto utilizando-se a espectrofotometria. Pesou-se, pulverizou-se e homogeneizou-se vinte comprimidos da amostra comercial. Transferiu-se quantidade do pó equivalente a 0,250 g de AAS para Erlenmeyer de $125 \mathrm{~mL}$ e adicionou-se $15 \mathrm{~mL}$ da solução de $\mathrm{NaOH}$ $0,500 \mathrm{~mol} \mathrm{~L}^{-1}$ (previamente padronizada com $\mathrm{HK}\left(\mathrm{C}_{8} \mathrm{H}_{4} \mathrm{O}_{4}\right)$ ). A solução contendo AAS e $\mathrm{NaOH}$ foi fervida cuidadosamente por 10 minutos e titulou-se o excesso de $\mathrm{NaOH}$ com a solução de $\mathrm{HCl}$ 0,500 $\mathrm{mol} \mathrm{L}^{-1}$ (previamente padronizada com $\mathrm{Na}_{2} \mathrm{CO}_{3}$ ), utilizando-se vermelho de fenol como indicador. Realizou-se ensaio em branco e efetuou-se as correções necessárias. Cada $\mathrm{mL}$ de $\mathrm{NaOH} 0,500 \mathrm{~mol} \mathrm{~L}^{-1}$ equivale a $45,040 \mathrm{mg}$ de AAS. A quantificação de AAS em amostras comerciais utilizando-se a titulação de retorno ácido-base foi realizada em triplicata. O procedimento descrito na Farmacopeia Brasileira foi adaptado com o objetivo de gerar uma quantidade menor de resíduo químico.

\section{Análises estatísticas}

A análise estatística dos dados foi realizada por meio de análise de variância (ANOVA fator único), testes te f com nível de confiança de 95\% (probabilidade inferior a 5\% - p < 0,05). A avaliação estatística dos resultados foi realizada por meio do Software MS Excel ${ }^{\circledR}$.

\section{RESULTADOS E DISCUSSÃO}

Para verificar a possibilidade de desenvolvimento de um método espectrofotométrico, para a quantificação de AAS em formulações farmacêuticas, foram obtidos os espectros UV (200-400 nm) da Figura 2 a partir de uma solução de AAS $10 \mu \mathrm{g} \mathrm{mL}^{-1} \mathrm{em} \mathrm{NaOH} 0,100 \mathrm{~mol}$ $\mathrm{L}^{-1}, \mathrm{HCl} 0,100 \mathrm{~mol} \mathrm{~L}^{-1}$ e etanol absoluto. Foram observadas duas bandas de absorção máxima em 217 e 297 nm em meio alcalino, em 230 e 276 em meio ácido e 227 e 275 nm em meio neutro. Com base nos espectros UV obtidos, analisando a altura e forma da banda de absorção, optou-se para o desenvolvimento de método analítico realizar as medidas espectrofotométricas em meio alcalino $(\mathrm{NaOH}$ 0,100 mol L-1) no comprimento de onda no máximo de absorção $\left(\lambda_{\text {max }}\right)$ de $297 \mathrm{~nm}$.

Na validação do método analítico para a quantificação de AAS em formulações farmacêuticas, foi realizado o estudo de seletividade por meio de análises dos espectros UV (Figura 3) obtidos para o AAS e os excipientes sacarina sódica e vanilina, solúveis em $\mathrm{NaOH}$ $0,100 \mathrm{~mol} \mathrm{~L}^{-1}$. Observa-se nos espectros UV que a sacarina não apresenta banda de absorção na faixa de comprimento de onda estudada.

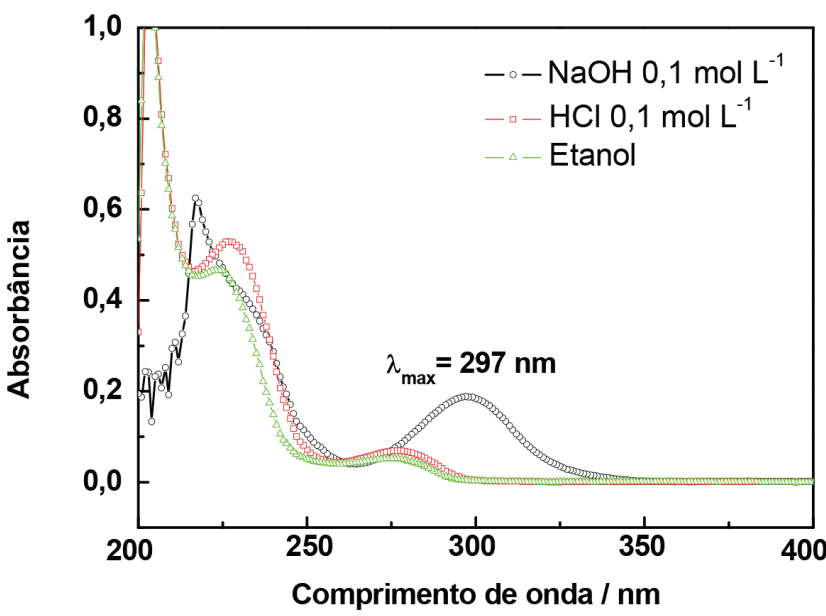

Figura 2. Espectros de absorção no UV do ácido acetilsalicílico

No entanto, a vanilina apresentou bandas de absorção com máximos em 250 e 347 nm, sem sobreposição em relação ao comprimento de onda máximo do AAS (297 nm). Observando-se o espectro UV para a mistura (AAS + vanilina + sacarina) notam-se dois máximos de absorção para o AAS e vanilina em 299 e 348 nm, respectivamente. Como base nas medidas espectrofotométricas, realizadas com os excipientes, acredita-se que em baixas contrações a vanilina não é um potencial interferente na análise das formulações farmacêuticas. Os demais excipientes, encontrados nas formulações farmacêuticas contendo AAS, como o fosfato de cálcio dibásico, a celulose e o amido, são insolúveis em $\mathrm{NaOH} 0,100 \mathrm{~mol} \mathrm{~L}^{-1}$ não interferindo na análise das formulações farmacêuticas.

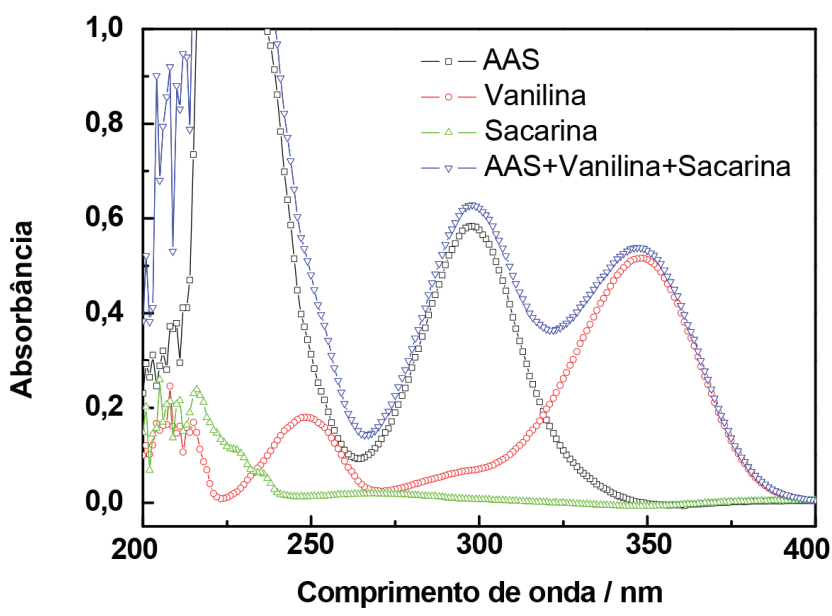

Figura 3. Espectro de absorção no UV do ácido acetilsalicílico e dos excipientes em NaOH 0,100 $\mathrm{mol} \mathrm{L}^{-1}$

Após o estudo de seletividade foram obtidos os espectros de absorção no UV para AAS, com a concentração variando de 24 a 36 $\mu \mathrm{g} \mathrm{mL} \mathrm{mL}^{-1}$ (de 80 - $120 \%$ da concentração teórica do teste), em NaOH $0,100 \mathrm{~mol} \mathrm{~L}^{-1}$ (Figura 4).

A curva analítica, obtida por meio do método dos mínimos quadrados, apresentou a equação $y=0,0188 x+0,0069$ e um coeficiente de correlação de 0,9994 , indicando a linearidade do método dentro dos limites de concentrações estudadas, além de estar de acordo com o critério mínimo aceitável do coeficiente de correlação $(\mathrm{r}=$ 0,990) descrito na Resolução para Validação de Métodos Analíticos da ANVISA (RDC nº 166 de 24/07/2017). ${ }^{23}$ Por meio da análise de variância, testou-se a significância estatística da curva ajustada e a linearidade do método (Tabela 1). No teste do ajuste da curva 


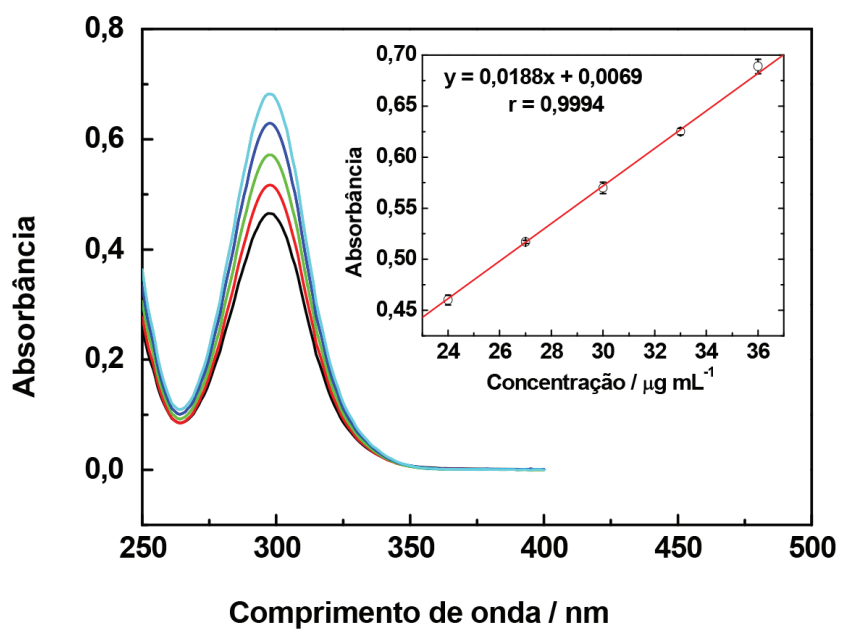

Figura 4. Espectros de absorção no UV obtidos para o AAS em $\mathrm{NaOH}$

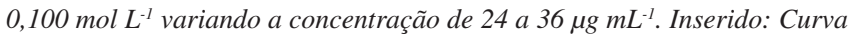
analítica

obteve-se um valor de $\mathrm{f}_{\text {calculado }}(1,926)$ menor que o $\mathrm{f}_{\text {tabelado }}(3,710)$, comprovando-se que não houve falta de ajuste na faixa de concentração estudada, a um nível de confiança de $95 \%$. Em seguida, a validade da regressão (linearidade) foi analisada por meio da comparação dos valores de $\mathrm{f}$ tabelado e calculado. $\mathrm{O}$ valor de $\mathrm{f}_{\text {calculado }}(3376,2)$ muito maior que o $\mathrm{f}_{\text {tabelado }}(4,670)$ indica que o método é linear, a um nível de confiança de $95 \%$.

Tabela 1. Tabela de análise de variância para o ajuste de um modelo pelo método dos mínimos quadrados

\begin{tabular}{lccccc}
\hline Fonte & SQ & GL & MQ & $\mathrm{f}_{\text {calculado }}$ & $\mathrm{f}_{\text {tabelado }}$ \\
\hline Modelo & $9,54 \times 10^{-2}$ & 1 & $9,54 \times 10^{-4}$ & 3376,2 & 4,670 \\
Residual & $3,67 \times 10^{-4}$ & 13 & $2,82 \times 10^{-5}$ & Curva linear \\
Falta de ajuste & $1,34 \times 10^{-4}$ & 3 & $4,48 \times 10^{-5}$ & 1,926 & 3,710 \\
Erro puro & $2,32 \times 10^{-4}$ & 10 & $2,32 \times 10^{-5}$ & Não há falta de ajuste \\
\hline Total & $9,62 \times 10^{-2}$ & 14 & $6,87 \times 10^{-3}$ & & \\
\hline
\end{tabular}

$\mathrm{SQ}=$ Soma Quadrática. $\mathrm{GL}=$ Graus de Liberdade. $\mathrm{MQ}=$ Média Quadrática.

A partir da curva analítica foram calculados os limites de detecção de $1,97 \mu \mathrm{g} \mathrm{mL} \mathrm{m}^{-1}$ e de quantificação de $6,57 \mu \mathrm{g} \mathrm{mL} \mathrm{m}^{-1}$, assegurando-se que o método proposto pode detectar e quantificar uma ampla faixa de concentração com segurança, podendo ser aplicado na detecção e quantificação de AAS em formulações farmacêuticas.

Em seguida, foram avaliadas a repetibilidade e a precisão intermediária por meio de medidas espectrofotométricas de soluções contendo AAS, em três níveis de concentração diferentes, em dois dias e com analistas diferentes. Os resultados obtidos para a repetibilidade e precisão intermediária, apresentados na Tabela 2, demonstram que o método proposto apresenta boa precisão com valores de CV menores que $2 \%$.

Tabela 2. Resultados de repetibilidade e de precisão intermediária obtidos por meio de medidas espectrofotométricas no UV de soluções contendo AAS em três níveis de concentração diferentes $\left(24,30\right.$ e $\left.36 \mu \mathrm{g} \mathrm{mL} L^{-1}\right)$

\begin{tabular}{ccccc}
\hline Dia & Analista & $\begin{array}{c}\text { Concentração } \\
\left(\mu \mathrm{g} \mathrm{mL}^{-1}\right)\end{array}$ & $\mathrm{CV}(\%)^{*}$ & $\mathrm{CV}(\%)^{* *}$ \\
\hline \multirow{2}{*}{1} & 24 & 1,45 & - \\
& \multirow{2}{*}{1} & 30 & 0,34 & - \\
& & 36 & 1,27 & - \\
\hline \multirow{2}{*}{2} & 24 & 1,20 & 1,26 \\
& & 30 & 0,44 & 1,31 \\
& & 36 & 0,56 & 0,90 \\
\hline
\end{tabular}

$\mathrm{CV}=$ Coeficiente de variação; *repetibilidade $(\mathrm{n}=3)$; **precisão intermediária $(n=6)$.

Com a confirmação da precisão do método proposto foram realizados ensaios para a determinação da exatidão, por meio do teste de adição de padrão de AAS, obtendo-se recuperações médias para três níveis diferentes de AAS (20 mg, $50 \mathrm{mg}$ e $70 \mathrm{mg}$ ) nas formulações farmacêuticas de referência e genérica iguais a 99,42\% $\pm 11,65$ e $95,94 \% \pm 5,46$, respectivamente. Os valores de $t_{\text {calculado }}(0,086-$ referência; 1,288 - genérico) são menores que o $t_{\text {tabelado }}(4,303)$, indicando que os valores de recuperação encontrados são estatisticamente iguais a $100 \%$, a um nível de confiança de $95 \%$, confirmando que não há interferência significativa da matriz (excipientes) nas amostras de formulações farmacêuticas analisadas (referência e genérico) e que o método proposto apresenta boa exatidão.

Com o objetivo de avaliar a robustez, capacidade do método proposto em resistir a pequenas variações dos parâmetros analíticos, verificou-se o comportamento dos resultados em relação a duas variáveis: marca do reagente utilizado no preparo da solução de $\mathrm{NaOH} 0,100 \mathrm{~mol} \mathrm{~L}^{-1} \mathrm{e}$ a temperatura do laboratório onda as medidas espectrofotométricas foram realizadas. Os resultados experimentais obtidos para a robustez são apresentados na Tabela 3.

A análise estatística dos dados obtidos, variando a marca do reagente utilizado no preparo da solução de $\mathrm{NaOH} 0,100 \mathrm{~mol} \mathrm{~L}^{-1}$, realizada por meio da ANOVA, demonstrou que mudando a marca do reagente ocorre uma influência significativa nos resultados, a um nível de confiança de $95 \%$, portanto, o método proposto não foi robusto nesse parâmetro avaliado. No entanto, essa variação não afeta nos resultados das análises em formulações farmacêuticas e, segundo a orientação sobre validação de métodos analíticos (DOQ-CGCRE-008, $5^{\text {a }}$ revisão, INMETRO), ${ }^{32}$ a robustez é um parâmetro opcional dentro dos estudos de validação. $\mathrm{O}$ teste $\mathrm{f}$ foi aplicado para comparar as precisões obtidas, empregando-se o método espectrofotométrico proposto em duas temperaturas diferentes do local da análise, resultando em um valor de $\mathrm{f}_{\text {calculado }}$ menor que o valor de $\mathrm{f}_{\text {tabelado }}$ para as temperaturas

Tabela 3. Resultados relativos à robustez do método proposto (Concentração de AAS $30 \mu \mathrm{g} \mathrm{mL}^{-1}$ )

\begin{tabular}{cccccc}
\hline \multicolumn{2}{c}{ Parâmetros variados } & $\begin{array}{c}\text { Concentração média } \\
\left(\mu \mathrm{mL}^{-1}\right)\end{array}$ & DP & CV $(\%)$ & ANOVA \\
\hline \multirow{3}{*}{ Marca do NaOH } & Reagen & 27,63 & 0,18 & 0,66 & 0,65 \\
& Synth & 27,38 & 0,18 & $\mathrm{f}_{\text {calculado }}=92,1 ; \mathrm{f}_{\text {tabelado }}=5,14$ \\
& Vetec & 30,29 & 0,43 & 1,43 & $\mathrm{f}_{\text {calculado }}=1,35 ; \mathrm{f}_{\text {tabelado }}=19,0$ \\
\multirow{2}{*}{ Temperatura } & $24^{\circ} \mathrm{C}$ & 30,03 & 0,50 & 1,65 & $\mathrm{t}_{\text {calculado }}=0,683 ; \mathrm{t}_{\text {tabelado }}=4,303$ \\
& $27^{\circ} \mathrm{C}$ & 30,29 & 0,43 & 1,43 &
\end{tabular}

$\mathrm{n}=3$. $\mathrm{DP}=$ Desvio padrão. $\mathrm{CV}=$ Coeficiente de variação. 
Tabela 4. Resultados obtidos nas análises de AAS em formulações farmacêuticas empregando-se os métodos espectrofotométrico proposto e o titulométrico de retorno ácido-base recomendado pela Farmacopeia Brasileira

\begin{tabular}{|c|c|c|c|c|c|}
\hline \multicolumn{4}{|c|}{ AAS (mg comprimido ${ }^{-1}$ ) } & \multicolumn{2}{|c|}{ Erro relativo (\%) } \\
\hline Amostras & Rótulo & Titulação de retorno ácido-base ${ }^{\mathrm{a}}$ & Método espectrofotométrico ${ }^{\mathrm{a}}$ & $\mathrm{ER}_{1}^{\mathrm{b}}$ & $\mathrm{ER}_{2}^{\mathrm{c}}$ \\
\hline Referência & 500 & $502 \pm 4$ & $499 \pm 7$ & $-0,59$ & $-0,20$ \\
\hline Genérico & 500 & $504 \pm 3$ & $497 \pm 7$ & $-1,38$ & $-0,60$ \\
\hline
\end{tabular}

${ }^{\mathrm{a}} \mathrm{n}=3 ;{ }^{\mathrm{b}} \mathrm{ER}_{1}=[$ (método espectrofotométrico - titulação de retorno ácido-base $) /$ titulação de retorno ácido-base $]$ x 100; ${ }^{\mathrm{c}} \mathrm{ER}_{2}=\left[\left(\right.\right.$ método espectrofotométrico $^{-}$ - valor no rótulo)/ valor no rótulo] x 100.

$24{ }^{\circ} \mathrm{C}$ e $27{ }^{\circ} \mathrm{C}$, indicando que não existe diferença significativa entre as precisões das duas análises, a um nível de confiança de $95 \%$, possibilitando a aplicação do teste $\mathrm{t}$. $\mathrm{O}$ valor de $\mathrm{t}_{\text {calculado }}$ é menor que o $t_{\text {tabelado }}$, indicando que não existem diferenças significativas, a um nível de confiança de $95 \%$, entre as médias dos resultados obtidos nas duas temperaturas $\left(24^{\circ} \mathrm{C}\right.$ e $\left.27^{\circ} \mathrm{C}\right)$ estudadas.

A Tabela 4 apresenta os resultados obtidos na quantificação de AAS em formulações farmacêuticas empregando-se os métodos titulométrico de retorno ácido-base, recomendado pela Farmacopeia Brasileira, ${ }^{15}$ e o espectrofotométrico.

A análise estatística de ANOVA demonstrou que não há diferença significativa entre os resultados obtidos pelos dois métodos empregados para as duas formulações farmacêuticas analisadas $\left(\mathrm{f}_{\text {calculado }}=\right.$ 0,$\left.87 ; \mathrm{f}_{\text {tabelado }}=4,06 ; \mathrm{p}<0,05\right)$. O teste $\mathrm{f}$ foi aplicado para comparar as precisões obtidas empregando-se os métodos titulométrico de retorno ácido-base e o espectrofotométrico, resultando em valores de $\mathrm{f}_{\text {calculado }}$ (3,06 - referência; 5,44 - genérico) menores que o valor de $\mathrm{f}_{\text {tabelado }}$ $(19,0)$, para as duas amostras de formulações farmacêuticas, indicando que não existe diferença significativa entre as precisões dos dois métodos empregados $(\mathrm{p}<0,05)$ possibilitando a aplicação do teste t. Os valores de $t_{\text {calculado }}(0,7894$ - referência; 1,952 - genérico) comparados com o $t_{\text {tabelado }}(4,303)$, indicaram que não existe diferença significativa $(\mathrm{p}<0,05)$ entre as médias dos resultados dos dois métodos para as duas formulações farmacêuticas analisadas. $O$ teste $t$ também foi aplicado para comparar as médias obtidas, empregando-se os métodos titulométrico de retorno ácido-base e o espectrofotométrico, com os valores descritos nos rótulos das formulações farmacêuticas analisadas. Os valores de $t_{\text {calculado }}$ para o método titulométrico de retorno ácido-base (0,8660 - referência; 2,309 - genérico) são menores que o $t_{\text {tabelado }}(4,303)$, indicando que os valores das médias obtidas por esse método são estatisticamente iguais a $500 \mathrm{mg} \mathrm{comprimido}^{-1}$ (p $<0,05$ ). Para o método espectrofotométrico proposto, os valores de $\mathrm{t}_{\text {calculado }}(0,2474$ - referência; 0,7423 - genérico) também são menores que o $t_{\text {tabelado }}(4,303)$, indicando que os valores das médias obtidas por esse método são estatisticamente iguais a $500 \mathrm{mg} \mathrm{comprimido}^{-1}$, em um nível de confiança de $95 \%$. Esses resultados demostraram que o método espectrofotométrico validado é uma excelente alternativa na quantificação de AAS em formulações farmacêuticas.

A prática sugerida para a disciplina de Análise Instrumental em cursos de graduação, utilizando-se o método espectrofotométrico no UV na quantificação de AAS em formulações farmacêuticas, realizada em cinco aulas práticas, mostrou-se simples, rápida, segura e de baixo custo e quando aplicada no laboratório de ensino proporcionou aos estudantes o contato com a técnica espectrofotométrica no UV, promovendo a discussão e a compreensão dessa técnica, de conceitos importantes envolvidos nos estudos de validação de métodos (seletividade, linearidade, intervalo, precisão, limite de detecção, limite de quantificação, exatidão e robustez) e da aplicação de testes estatísticos.

\section{CONCLUSÃO}

A prática proposta de validação de método espectrofotométrico de análise para a quantificação de AAS foi utilizada como ferramenta metodológica, favorecendo o processo de ensino-aprendizagem e ocasionando um ganho pedagógico para os estudantes da disciplina de Análise Instrumental. O método espectrofotométrico na região do UV validado mostrou-se específico, linear, preciso, exato, com baixos limites de detecção e quantificação, viabilizando o seu emprego na quantificação de AAS em formulações farmacêuticas, em análises de rotina de controle de qualidade de comprimidos e atividade prática em laboratórios de ensino, pois é um método simples, rápido, seguro e de baixo custo.

\section{MATERIAL SUPLEMENTAR}

Os testes estáticos, a explicação detalhada dos parâmetros de validação e os procedimentos das padronizações das soluções de $\mathrm{NaOH}$ e $\mathrm{HCl}$ e da quantificação de AAS em comprimidos estão disponíveis em http://quimicanova.sbq.org.br, em arquivo pdf, com livre acesso.

\section{AGRADECIMENTOS}

Ao apoio financeiro da Universidade Católica de Brasília (UCB).

\section{REFERÊNCIAS}

1. Athota, R. V.; Jagarlapudi, S. K.; Singampalli, M. R.; J. Appl. Pharm. Sci. 2017, 7, 48

2. Alfonso, L.; Ai, G.; Spitale, R. C.; Bhat, G. J.; Br. J. Cancer 2014, 111, 61.

3. Cadavid, A. P.; Front. Immunol. 2017, 8, 1.

4. Sena, M. M.; Fernandes, J. C. B.; Rover Jr., L.; Poppi, R. J.; Kubota, L. T.; Anal. Chim. Acta 2000, 409, 159.

5. Kokot, Z.; Burda, K.; J. Pharm. Biomed. Anal. 1998, 18, 871.

6. Bouhsain, Z.; Garrigues, S.; de la Guardia, M.; Fresenius'J. Anal. Chem. 1997, 357, 973

7. Karim, M. M.; Jeon, C. W.; Lee, H. S.; Alam, S. M.; Choi, J. H.; Jin, S. O.; Das, A. K.; J. Fluoresc. 2006, 16, 713.

8. Navalón, A.; Blanc, R.; del Olmo, M.; Vilchez, J. L.; Talanta 1999, 48, 469.

9. Shinde, S. S.; Kachave, R. N.; Chaudhari, S. R.; Int. J. Pharm. Res. 2013, 5, 19 .

10. Franeta, J.T.; Agbaba, D.; Eric, S.; Pavkov, S.; Aleksic, M.; Vladimirov, S.; Il Farmaco 2002, 57, 709.

11. Rover Jr., L.; Garcia, C. A. B.; Neto, G. O.; Kubota, L. T.; Galembeck, F.; Anal. Chim. Acta 1998, 366, 103.

12. Torriero, A. A. J.; Luco, J. M.; Sereno, L.; Raba, J.; Talanta 2004, 62, 247.

13. Park, J.; Eun, C.; Electrochim. Acta 2016, 194, 346.

14. Iacob, A.; Manea, F.; Vaszilcsin, N.; Picken, S. J.; Schoonman, J.; Int. J. Electrochem. Sci. 2015, 10, 5661.

15. Farmacopeia Brasileira, $5^{\mathrm{a}}$ ed., Agência Nacional de Vigilância Sanitária: Brasília, 2010, p. 863.

16. Borba, P. A. A.; Riekes, M. K.; Pereira, R. N.; Stulzer, H. K.; Dalla Vecchia, D.; Quim. Nova 2013, 36, 582. 
17. Alves, L. D. S.; Rolim, L. A.; Fontes, D. A. F.; Rolim-Neto, P. J.; Soares, M. F. de L. R.; Sobrinho, J. L. S.; Quim. Nova 2010, 33, 1967.

18. Polonini, H. C.; dos Santos, F. C.; Vaz, U. P.; Brandão, M. A. F.; Raposo, N. R. B.; Quim. Nova 2011, 34, 516.

19. Barboza, F. M.; Dalla Vecchia, D.; Pereira, A. V.; Stulzer, H. K.; Silva, M. A. S.; Quim. Nova 2010, 33, 747.

20. do Rêgo, J. F.; de Moura, J. I.; Moita, G. C.; Quim. Nova 2010, 33, 471.

21. de Moura, J. I.; Moita, G. C.; Quim. Nova 2012, 35, 627.

22. Goes Junior, E. J. A.; Roeder, J. S.; Oliveira, K. B. L.; Ferreira, M. P.; Silva, J. G.; Rev. Virtual Quim. 2017, 9, 1747.

23. Agência Nacional de Vigilância Sanitária (ANVISA); $R D C N^{\circ} 166$, de 24/07/2017.

24. Simoni, D. A.; Andrade, J. C.; Simoni, J. A.; Quim. Nova 2011, 34, 1818.

25. Galiazzi, M. C.; Gonçalves, F. P.; Quim. Nova 2004, 27, 326.

26. da Luz Júnior, G. E.; de Sousa, S. A. A.; Moita, G. C.; Moita Neto, J. M.; Quim. Nova 2004, 27, 164.
27. Silva, J. G.; Lehmkuhl, A.; Alcanfor, S. K. B.; Quim. Nova 2009, 32, 1055.

28. Alcanfor, S. K. B.; Zara, L. F.; Pinheiro, L. A.; Faria, J. M.; Silva, J. G.; Educ. Quim. 2010, 21, 202.

29. Sousa, A. G.; Chagas, F. W. M.; Gois, 1. C.; Silva, J. G.; Rev. Virtual Quim. 2018, 10, 502.

30. ICH - The International Conference on Harmonisation of Technical Requirements for Registration of Pharmaceuticals for Human Use; $Q$ 2B-validation of Analytical procedure: methodology, 1996.

31. Mendham, J.; Denney, R. C.; Barnes, J. D.; Thomas, M. J. K.; Análise Química Quantitativa, 6a ed., LTC: Rio de Janeiro, 2002.

32. Instituto Nacional de Metrologia, Normalização e Qualidade Industrial (INMETRO); Orientação sobre Validação de Métodos Analíticos, DOQCGCRE-008, $5^{\text {a }}$ revisão, 2016. 